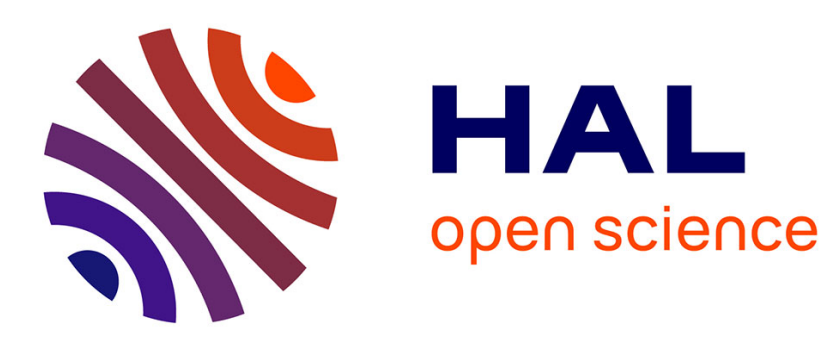

\title{
Resilience Assessment and Importance Measure for Interdependent Critical Infrastructures
}

Xing Liu, Yiping Fang, Elisa Ferrario, Enrico Zio

\section{To cite this version:}

Xing Liu, Yiping Fang, Elisa Ferrario, Enrico Zio. Resilience Assessment and Importance Measure for Interdependent Critical Infrastructures. ASCE-ASME Journal of Risk and Uncertainty in Engineering Systems, Part B: Mechanical Engineering, 2021, 10.1115/1.4051196 . hal-03226806

\section{HAL Id: hal-03226806 https://hal.science/hal-03226806}

Submitted on 15 May 2021

HAL is a multi-disciplinary open access archive for the deposit and dissemination of scientific research documents, whether they are published or not. The documents may come from teaching and research institutions in France or abroad, or from public or private research centers.
L'archive ouverte pluridisciplinaire HAL, est destinée au dépôt et à la diffusion de documents scientifiques de niveau recherche, publiés ou non, émanant des établissements d'enseignement et de recherche français ou étrangers, des laboratoires publics ou privés. 


\title{
Resilience Assessment and Importance Measure for Interdepend- ent Critical Infrastructures
}

\author{
Xing Liu, Yi-Ping Fang* \\ Chair Risk and Resilience of Complex Systems \\ Laboratoire Génie Industriel, CentraleSupélec, Université Paris-Saclay \\ 3 Rue Joliot Curie, 91190 Gif-sur-Yvette, France \\ E-mail: yiping.fang@centralesupelec.fr
}

Elisa Ferrario

Research Center for Integrated Disaster Risk Management (CIGIDEN), ANID/FONDAP/15110017, Avenida Vicuña Mackenna 4860, Santiago, Chile

E-mail: elisa.ferrario@cigiden.cl

\section{Enrico Zio}

Centre for Research on Risk and Crises (CRC), Mines ParisTech Sophia Antipolis, PSL Research University, 06904 Sophia Antipolis, France;

Dipartimento di Energia - Politecnico di Milano, Via Ponzio 34/3, 20133 Milano, Italy

E-mail: enrico.zio@polimi.it

\begin{abstract}
Based upon a novel control-based dynamic modelling framework, this paper proposes two new indicators, i.e., resilience by mitigation and resilience by recovery, for the resilience analysis of interdependent critical infrastructures (ICIs) under disruptions. The former is built from the protection activities before and during the mitigation phase of a disruptive event, and the latter is the result of the restoration efforts which take place at the recovery phase. The total resilience of ICIs combines both of these two aspects by taking into account the preferences of the decision-makers. We demonstrate the applicability of the proposed modelling framework and metrics in a case study involving ICIs made of a power grid and a gas distribution system. Owing to the new resilience indicators, the priorities of subsystems and links within ICIs at different phases can be ranked, therefore different resilience strategies at different phases of disrup-
\end{abstract}

\footnotetext{
•Corresponding author: yiping.fang@centralesupelec.fr
} 
tive events are compared. The results show that proposed metrics can be used by stakeholders of ICIs on improving the effectiveness of system protection measurements.

Keywords: Critical infrastructure, System resilience, Resilience metrics, Dynamic modelling, Model Predictive Control, Importance measure, Global sensitivity analysis

\section{Introduction}

Critical infrastructures (CIs) are complex systems that provide essential commodities, e.g., energy, natural gas, water, etc., and services e.g., telecommunication, banking, healthcare, etc., for the welfare of modern society and life quality of people. These CIs are exposed to various types of hazards, e.g., cyber incidents, natural disasters, technical failures and acts of terrorism [1-3].

With the increasing complexity of the system functions, physical and functional dependencies among diverse CIs are simultaneously growing. These dependency relationships improve the operation efficiency of interdependent CIs (ICIs), but also increase system vulnerability, e.g., provoking cascading failures, which is the situation where an initial failure originated in one CI propagates to other CIs and finally results in efficiency reduction, function loss or destruction of the entire system-of-systems [4-7]. In recent years, diverse severe disruptive events occurred to ICIs around the world and caused immense economic losses and serious casualties, e.g., 9/11 terrorist attack, 2003 country-wide blackout in Italy, 2010 Chilean earthquake and tsunami, 2011 Tohoku earthquake in Japan, 2017 Harvey hurricane in North America, etc. Facing these catastrophic events, great emphasis has been placed on studies of system resilience [8-10].

Many definitions of system resilience have been proposed from various perspectives [11]. Although there is still vagueness regarding the concept of system resilience, its two main aspects are universally recognized: 1) the resistance capacity, or the complement of vulnerability, which is the capacity of the system to maintain the nominal operations and mitigate the negative impacts of disruptions, and 2) the recovery capacity, or recoverability, which is the capacity of a system to bound back to the operational state following a risk source or an adverse event. Therefore, we define the system resilience for ICIs as the capacities of a system to resist to and 
to recover from disruptive events.

In order to enable the development of resilient systems, many system resilience metrics have been proposed $[11,12]$. Relying on the application of interest, the system resilience metrics could be classified according to different criteria, such as deterministic/probabilistic, dynamic/static, and/or integration-based/ratio-based, etc. $[13,14]$. One classical resilience metric is an integration-based method introduced in [15]. This method takes into account both the magnitude and duration of deviation of actual system performance from targeted system performance during a disruptive event. It is effective to assess and compare system resilience in different situations, e.g., system resilience design, system resilience improvement, system resilience examination, and inspire the development of other resilience assessment and analysis approaches [14]. For example, Ouyang et al. [16] introduced a probabilistic and static annual resilience measure, which provides a statistical result of estimated system resilience under multiple potential disruptions within a suitable length of time. Fang et al. [17] proposed a dynamic resilience metric that takes into account the lowest level of system performance and is able to monitor the evolution of system resilience at each time step. In the context of ICIs, Nan and Sansavini [18] presented an integrated metric for interdependent system resilience quantification that considers multiples phases of disruptive events. Kong and Simonovic [19] proposed a probabilistic metric based on the expansion of a single hazard deterministic resilience model to multiple hazard scenarios.

Unlike ecological systems, whose resilience is an inherent and spontaneous feature, the resilience of engineering systems, e.g., ICIs, is the result of appropriate protection activities, i.e., planning and resource allocation $[11,20]$. The protection activities are implemented in different phases during the disruptive events, for different purposes and executed by different operators [21]. Before the onset of an adverse event or activated at the beginning of the failure propagation phase, i.e., the mitigation phase, pre-event preparedness or mitigation activities are arranged to minimize the impacts of the disruption and extenuate the consequences of failure [22], such as enhancing the strength of elements, implementing redundancies, adjusting system topology and other self-healing mechanisms [16]. Recovery activities are post-event measures, such as repair- 
ing malfunctioned elements, replacing damaged elements, retrofitting structures, etc. They are implemented after the disruptive event, sometimes when system performance reaches a stable disrupted level, such as repair or replacement of failed components, retrofitting and reconstruction $[23,24]$.

Many existing works of system resilience focus on the recovery aspect [11, 25, 26]. Although some resilience analysis and measurement approaches attempt to include the entire failurerecovery process of the disturbed system, few of them differentiate the contributions of mitigation activities and recovery activities. In fact, there is the necessity to measure both two aspects of system resilience as the results of resilience assessment affect the effectiveness of resilience improvement strategies. For example, resilience metric is combined into the importance measure approach to prioritize the system elements [17]. However, the priorities of important system elements and key activities affecting system resilience may evolve during the disruptive event. For example, failure mitigation of a nuclear power plant within an energy system should have very high priority, as its failure leads to severe consequences, but in the recovery phase, once the core components are well protected, the less prioritized components in the first phase become important, i.e., the electrical links connected to users also should be highly prioritized, in order to resume electricity supply rapidly. Nevertheless, with the current system resilience assessments, it is difficult to capture the change on the criticality of system elements and to provide specific insights on resilience actions in the different phases of a disruptive event.

To address the above issue, this paper proposes generic resilience metrics which highlights the contributions of both mitigation and recovery aspects. Taking into account the characteristics of ICIs, we first evaluate the system performance based on the resilience analysis framework proposed in [27]. The failure-recovery model of system elements and system parameters affecting system resilience are then defined. We propose a set of resilience indicators, including: 1) resilience by mitigation $R_{m}$, which corresponds to the capacity of resistance of the system after the disruptions; this depends on the protective measures implemented; and 2) resilience by recovery $R_{r}$, which refers to the capacity of recovery and it is due to the timeliness and rapidity of resto- 
ration actions, and 3) total resilience $R_{t}$, which evaluates the overall resilient performance of a system with the preference of decision-makers on mitigation and recovery aspects. A global sensitivity analysis-based importance measure approach is chosen to identify the most critical system parameters with respect to system resilience and to suggest the system resilience strategies in different phases of a disruptive event. Finally, this approach is illustrated by a case study, which consists of two interdependent CIs: a gas network and an electric power system. We compare the improvements of system resilience under the guidelines gained from the proposed approach and from a classical approach. The advantages and limitations of the proposed resilience metric are discussed.

The rest of the paper is organized as follows. Section II introduces the ICI modelling framework, discusses the failure-recovery mechanisms on ICI and defines related system parameters. In Section III, the generic resilience indicators are proposed and represented in terms of the system dynamic performance function. The operative steps of the distribution-based global sensitivity analysis are also introduced. Section IV applies the proposed resilience assessment and importance measures on a case study. The results obtained from the proposed approach and from a classical approach are compared. Section V concludes the work and future perspective.

\section{Optimization-based modelling framework}

In this Section, we introduce a modelling framework for ICIs, which is able to address the diversity of flows within different types of CIs and handle the constraints on system states. In the modelling framework, the system behaviour in the nominal operation mode and the failure mode is supposed to be under control and described by a set of dynamic equations. The flow distribution on ICIs is formulated and solved by Model Predictive Control (MPC) algorithm. Then we discuss the failure-recovery mechanism of system elements and introduce the system parameters affecting system resilience.

\subsection{Network representation of ICIs}

The functionality of ICIs relies on the exchanges of the different types of resources, which is tangible commodities or intangible services within it. Taking advantage of network theory, we 
represent the structure of the ICIs by a directed graph, as shown in Fig. 1. The nodes in the graph represent the subsystems in ICIs, which are components or groups of components [28]. Various flows within ICIs are produced, consumed, stored and transformed by the subsystems. The links in the network are tangible connections, i.e., gas pipelines, or intangible connections, i.e., logical dependency, between the nodes.

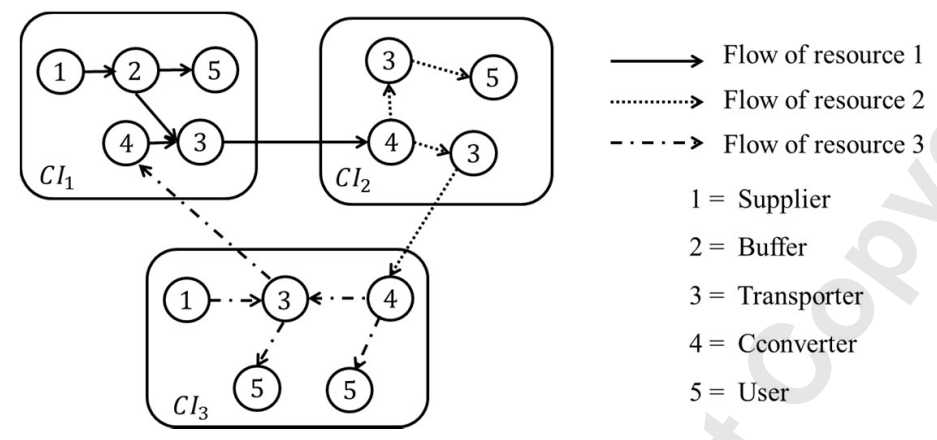

Fig. 1. A classical structure of ICIs

Fig. 1 shows a classical structure of ICIs. The subsystems within ICIs are classified into five common roles, i.e., suppliers, users, transporters, converters, and buffers. Suppliers are the sources of resource or service, e.g., renewable energy generators, water treatment plants, natural gas processing facilities. Users are the sinks in the networks, which consume the resources and absorb the flow, e.g., end-users of electricity, gas, water, etc. Transporters are the subsystems distributing the flows received from the precedent subsystems, e.g., substations of the power grid, compressor stations of a gas transmission network, etc. Converters are the subsystems transforming one type of resource or service to another, e.g., fossil-fuel power station, etc. Buffers are the subsystems with storage, e.g., batteries in power grids, gas reservoirs in the gas transmission network, etc.

\subsection{Control-based dynamic model}

Depending on the roles of each node, we consider input flow, output flow and/or storage level for them and the links associated with them. The output flow of the links downstream buffer nodes and the system driver nodes [29] are designated as system control inputs to guarantee system controllability. The following state-space model is used to model the evolution of system 
response under control:

$$
\begin{gathered}
x(t+1)=A x(t)+B u(t)+d(t), \\
y(t)=C x(t)
\end{gathered}
$$

where $x=\left[x_{1} \ldots x_{N_{x}}\right]^{\prime} \in R^{N_{x}}$ is the vector of the system states, which include the storage levels of buffer nodes, and output flow levels of links. $u=\left[u_{1} \ldots u_{N_{u}}\right]^{\prime} \in R^{N_{u}}$ is the vector of the control variables. $y=\left[y_{1} \ldots y_{N_{y}}\right]^{\prime} \in R^{N_{y}}$ is the vector of the system outputs, which is the actual level of the flows received by users. $d=\left[d_{1} \ldots d_{N_{d}}\right]^{\prime} \in R^{N_{d}}$ is the vector of disturbance variables associated with the losses of the system states due to the disruptions. The $N_{x} \times N_{x}$ matrix $A$ and the $N_{u} \times N_{x}\left(N_{u} \leq N_{x}\right)$ matrix $B$ contains the information of the system's connectivity and the transmission coefficients of flows.

The values of system states and control variables are typically limited by the capacities of the nodes and the links. The constraints are formulated as follows:

$$
\begin{aligned}
& 0 \leq x(t) \leq C P_{x}, \\
& 0 \leq u(t) \leq C P_{u},
\end{aligned}
$$

where the elements in $C P_{x}$ and $C P_{u}$ take values of the capacities of corresponding nodes or links. More details will be introduced in the next subsection.

The readers requiring more information about ICIs modelling framework please refer to [27].

\subsection{Failure-recovery mechanism and system parameters}

To describe the response of a system in the failure scenarios, we conclude a typical microscopic process of failure and recovery, i.e., failure-recovery mechanism of a system element (a node or a link), and define some system parameters in both time and system elements capacities dimensions in the aftermath of disruption. Fig. 2 shows the failure-recovery curve of a failed element $i$.

In nominal operation mode, the capacity of an element, which is the maximum of its states, remains as a fixed value. We denote $C P_{i}^{N}$ as the nominal capacity of $i$. 


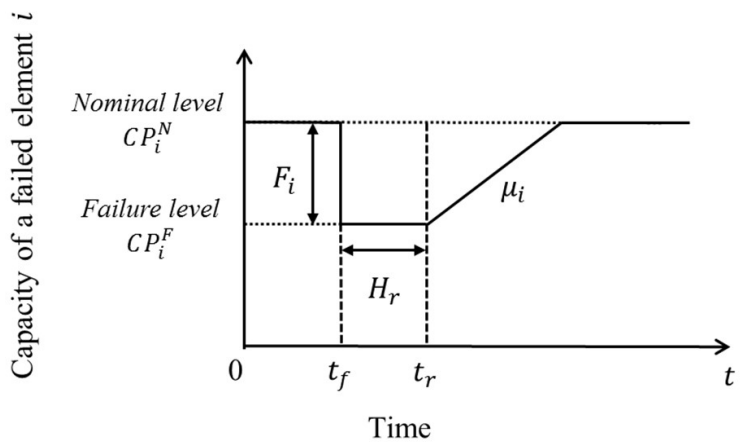

Fig. 2. The evolution of the capacity of a failed component in ICIs.

At time $t_{f}$, the stress of an initiating disruptive event hits element $i$ and results in the failure of element $i$ of the ICIs, i.e., a subsystem or a link. As a consequence of the element failure, its nominal capacity, e.g., $C P_{i}^{N}$, turns to a degradation state with reduction level $F_{i}$. Note that $F_{i}$ could be as large as $C P_{i}^{N}$, i.e., element $i$ is failed completely. The failure process at component level is usually very short compared to the time required for its restoration, and therefore it is regarded as instantaneous in Fig. 2. Then, we define response time $H_{r}$ to reflect the rapidity of the failure detection and preparedness of emergency actions of the system [30]. After the response time, i.e., at time instant $t_{r}=t_{f}+H_{r}$, element $i$ starts to be recovered. In some system models, the component state is a binary, i.e., it is in either function mode or failure mode, a failed component is supposed to be able to restore immediately after the execution of recovery activities. Owing to the continuous component state in our model, we are able to consider a more complex failure and recovery process for the elements in ICIs. For example, the recovery process of a failed element $i$ follows a linear function with a maintenance rate $\mu_{i}$, which relies on the effectiveness of recovery activities. Therefore, the capacity of element $i$ during the disruptive event, $C P_{i}$, can be represented as:

$$
C P_{i}(t)=C P_{i}^{N}-F_{i}+\mu_{i}\left(t-t_{r}\right)
$$

where

$$
F_{i}=\left\{\begin{array}{cc}
0 & \text { for } 0 \leq t<t_{f} \\
\text { magnitude of failure } & \text { for } t \geq t_{f}
\end{array},\right.
$$




$$
\mu_{i}=\left\{\begin{array}{cc}
0 & \text { for } 0 \leq t<t_{r} \\
\text { recovery rate } & \text { for } t \geq t_{r}
\end{array}\right.
$$

The capacities of system elements are expected to reach their original levels after the restoration activities, i.e., $C P_{i}(t) \leq C P_{i}^{N}$. In fact, depending on the applications we can consider more complex recovery functions to describe the component recovery process under the current modelling framework. In fact, the performance restoration speed of some complex components varies with time, for example, the recovery speed of components in freight system goes slower as slower, as the easy part will be recovered at first and the hard part will be recovered later [31]. Depending on the application and complexity of modelling, nonlinear functions can also be taken into account and accommodate to our modelling framework. For the sake of simplicity, we use a linear form for the recovery function of failed devices in the following of this work.

Once the restoration actions are implemented in the failed elements, i.e., from the instant $t_{r}$, the system performance will gradually increase. We define the time interval $H_{h}$ as the maximum time interval considered acceptable to restore the entire system, i.e., the system is expected to return to the nominal state before the instant $t_{h}=t_{f}+H_{h}$.

In the case of disruption, the disturbances result in malfunction or damage of some elements. Due to the physical connections and functional dependencies among the elements, there are two possible types of propagated failures that occurred in ICIs: 1) the incapability or incapable failure, that is caused by the structural or functional damages of elements, due to external disturbance or dependency on others. In this case, the capacity of the element reduces; 2) the inoperability, or inoperable failure, that results from the stop of upstreaming flow supply. In this case, the capacity of the element remains in a nominal level.

Hereafter, we focus on incapable failure cases, as the restoration activities are implemented on the incapable elements, and not on the inoperable elements. For the latter, they continue functioning immediately after the restoration of the elements that they depend on.

\subsection{Solution by MPC algorithm}

Taking into account the evolution in resource supplies due to renewable energy technologies and 
variances in user demands, different control strategies can be applied to distribute the resources. We embrace the Model Predictive Control (MPC) algorithm, which has been widely applied in CIs, such as in traffic control, resources dispatching and energy scheduling problems [32]. The classical MPC approach realizes a finite-horizon optimization by determining sequences of system states and control operations over a prediction horizon $N_{q}$ for the minimization of the objective function at each time step within $N_{q}$, and, then, implementing only the first control action [33]. If $N_{q}$ is large enough, the varying demands of users can be fulfilled, as the allocation of resources is regulated in advance. Notice that many variants exist in the literature besides the classical MPC scheme. For instance, nonlinear MPC can be used for multi-variable nonlinear systems with large delays and strong perturbations, although at the expense of computational complexity [34]; explicit MPC is able to compute the optimal control action offline by exploiting the parametric programming technique and is suited to control systems with fast dynamics requiring fewer run-time computations [35]; robust variants of MPC [36] seek to design controllers that maintain stability and performance despite model inaccuracies or uncertainties, e.g., by adopting the min-max MPC formulation. Interested readers can refer to [37] for a comprehensive discussion and review on the developments of MPC. In this study, we use the classical MPC method for the sake of conceptual simplicity and computational efficiency.

Considering that the units of the resources in ICIs are different, we represent the level of relative insufficiency of user $i_{y}$, by a normalized and non-dimensional variable :

$$
Y_{i_{y}}(t)=\frac{D_{i_{y}}(t)-y_{i_{y}}(t)}{D_{i_{y}}(t)} .
$$

Based on our dynamic model, the objective function is formulated to minimize the weighted sum of insufficiency function $Y_{i_{y}}(t)$ of users $i_{y} \in V_{u}$, within a predictive horizon $N_{q}$ :

$$
\min \sum_{q=0}^{N_{q}}\left(\sum_{i_{y} \in N_{y}} \omega_{i_{y}} Y_{i_{y}}(t+q \mid t)\right),
$$

where, $\omega_{i_{y}}$ is the weight assigned to the user $i_{y}$, and $\sum_{i_{y} \in V_{y}} \omega_{i_{y}}=1$. The objective function is 
subject to the constraints in Section 2.2 .

By solving the optimization problem with MPC, the control action $u(t \mid t)$ is obtained from the control sequence:

$$
u \triangleq\left\{u(t \mid t), u(t+1 \mid t), \ldots, u\left(t+N_{q}-1 \mid t\right)\right\}
$$

as a result of the optimization problem. Then, only the first control action $u(t \mid t)$ will be introduced in the recursion to calculate the system states at $t+1$.

\section{Resilience metric and importance measure}

\subsection{Generic resilience metric with three indicators}

As introduced before, there are various metrics proposed in the literature for the quantification of infrastructure system resilience. The main differences in the various resilience definitions and metrics stand in the way the capability to face adverse events is considered and measured, e.g., with respect to the time needed to recover system functionalities, to the down time during which urban services are not provided, to the number of citizens reallocated, to the urban efficiency loss, and so forth [18-20, 24]. Nevertheless, all represent aspects directly related to system functionality and to the ability to guarantee continuity of service, even when the global situation is compromised.

The system performance curve in relation to the system resilience property is typically triangular $[15,38,39]$ or trapezoid $[12,16,40]$, the former being representative of a system failure process which is almost instantaneous (e.g., following an earthquake) and the latter explicitly representing the process of gradual system performance degradation. Here we adopt the latter representation for ICIs as shown in Fig. 3, where the y-axis represents the performance function of a system, $P(t)$ and its level in nominal conditions is represented by a reference function $P R(t)$ (usually regarded as constant). Notice that Fig. 3 is different from the component level capacity degradation illustrated in Fig. 2. 


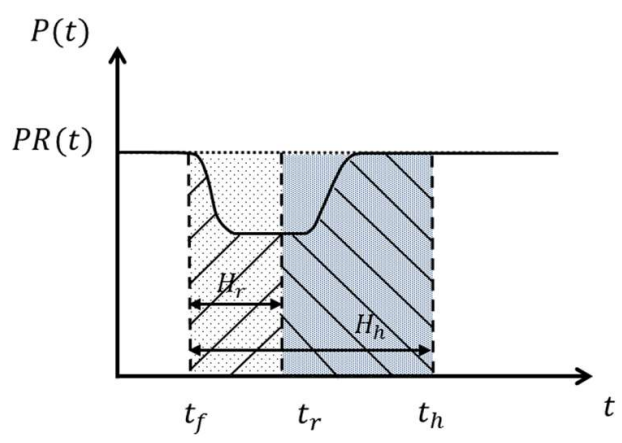

Fig. 3. System performance curves following the occurrence of an adverse event.

A representative method to evaluate system resilience based on the system performance curve is performance-integration resilience metrics $[18,41,42]$. The essence of this method is to evaluate the difference between the two system performance curves, i.e., expected system performance and actual system performance, or the system performance with original resilience strategies and with improved resilience strategies, over the time horizon of a disruptive event. This method is suitable for various applications and able to include the statistical properties of the randomness on the disruptions and system parameters [14].

In this work, we following the line of [8] and extend the basic performance-integration resilience metric to a set of resilience indicators for ICIs including: 1) resilience by mitigation $R_{m}$, which corresponds to the capacity of resistance of the system after the disruptions and depends on the protective activities implemented; and 2) resilience by recovery $R_{r}$, which refers to the capacity of recovery and it is due to the restoration strategies.

The resilience by mitigation is quantified as the proportion of the total area between actual system performance function $P(t)$ and the time axis (the area shaded with upward diagonal stripes in Fig. 3), to the total area between performance reference function $P R(t)$ and the time axis, for the time period $t_{f} \leq t \leq t_{r}$, which corresponds to the response time $H_{r}=t_{r}-t_{f}$, i.e., within the mitigation phase:

$$
R_{m}=\frac{\int_{t_{f}}^{t_{r}} P(t) d t}{\int_{t_{f}}^{t_{r}} P R(t) d t} .
$$


Similarly, the resilience by recovery is the proportion of the total area between actual system performance function $P(t)$ and the time axis (the area shaded with downward diagonal stripes in Fig. 3), to the area between performance reference function $P R(t)$ and the time axis, for the time period $t_{r} \leq t \leq t_{h}$ with $t_{h} \geq t_{r}$, i.e., from the start of restoration to the end of time horizon, $H_{h}-H_{r}=t_{h}-t_{r}$, i.e., within the recovery phase:

$$
R_{r}=\frac{\int_{t_{r}}^{t_{h}} P(t) d t}{\int_{t_{r}}^{t_{h}} P R(t) d t}
$$

Besides the two resilience indicators above, the overall level of system resilience, i.e., the total resilience $R_{t}$, is illustrated as the combination of two aspects, i.e., the weighted sum of resilience by mitigation and resilience by recovery:

$$
R_{t}=\omega_{m} R_{m}+\omega_{r} R_{r}
$$

with both $\omega_{r}$ and $\omega_{m}$ nonnegative and $\omega_{m}+\omega_{r}=1$. The definition of the weights depends on the preference of decision-makers [43].

In this work, we consider the proportion of the time period of two phases, as the weights:

$$
\begin{gathered}
\omega_{m}=\frac{H_{r}}{H_{h}}=\frac{t_{r}-t_{f}}{t_{h}-t_{f}}, \\
\omega_{r}=\frac{H_{h}-H_{r}}{H_{h}}=\frac{t_{h}-t_{r}}{t_{h}-t_{f}} .
\end{gathered}
$$

Therefore, the total resilience $R_{t}$ is represented as:

$$
R_{t}=\frac{t_{r}-t_{f}}{t_{h}-t_{f}} \frac{\int_{t_{f}}^{t_{r}} P(t) d t}{\int_{t_{f}}^{t_{r}} P R(t) d t}+\frac{t_{h}-t_{r}}{t_{h}-t_{f}} \frac{\int_{t_{r}}^{t_{h}} P(t) d t}{\int_{t_{r}}^{t_{h}} P R(t) d t} .
$$

In some cases where the preference system performance is fixed or approximately fixed over time, i.e., $P R(t)=P R$, the function of $R_{t}$ are simplified as:

$$
R_{t}=\frac{\int_{t_{f}}^{t_{h}} P(t) d t}{\int_{t_{f}}^{t_{h}} P R(t) d t}=\frac{\int_{t_{f}}^{t_{h}} P(t) d t}{P R\left(t_{h}-t_{f}\right)},
$$


which is the proportion of the total area between the actual system performance function $P(t)$ and the time axis (the dark area in Fig. 3), to the square area between the performance reference function $P R(t)$ and the time axis, for the time period $t_{f} \leq t \leq t_{h}$ with $t_{h} \geq t_{f}$, as shown in Fig. 3 .

By definition, the indicators of the resilience by mitigation, the resilience by recovery and the total resilience are measured as the proportion of lost area, so that take value in the interval $[0$, 1]. When the system remains fully operable under the disruptive event, $R_{m}=R_{r}=R_{t}=1$. The cases of $R_{m}=0, R_{r}=0$, or $R_{t}=0$ indicate extreme situations where the mitigation activities, restoration activities, or both of them are lacked or fail completely and the functionalities of ICIs are entirely interrupted from the moment where the initial failure occurs to the end of the time horizon.

\subsection{Resilience metric within ICIs dynamic modelling framework}

Note that in the generic resilience metric, the performance function $P(t)$ of a system can be any quantity of interest. For ICIs, the system performance can be defined from different viewpoints (reliability, availability, safety, economics, etc.), e.g., counting the number of operating components [38], the economic loss associated with the components and the casualties of people during the disaster [16]. The ICIs aim at providing stable and reliable services to users, and the measure of infrastructure resilience is how they enable and enhance daily life [11]. Given these goals of ICIs, we evaluate time-dependent system performance for ICIs in terms of the relative satisfactory level of users.

Based on the system dynamic model previously introduced, the actual performance function of ICIs, $P(t)$, is characterized directly in terms of the weighted sum of the complement of insufficiency function $Y_{i_{y}}$ :

$$
P(t)=\sum_{i_{y}}^{i_{y}=N_{y}} \omega_{i_{y}}\left(1-Y_{i_{y}}(t)\right)=\sum_{i_{y}}^{i_{y}=N_{y}} \omega_{i_{y}} \frac{y_{i_{y}}(t)}{D_{i_{y}}}
$$

where, $\omega_{i_{y}}$ with $i_{y}=1, \ldots, N_{y}$ is the weight of the $\mathrm{i}_{\mathrm{y}}$ th user. 
The nominal system performance of ICIs, $P R(t)$, is the normalized performance reference function under nominal operating conditions, which is the normalized total demand of resources or service by the users, therefore it is fixed value 1 :

$$
P R(t)=\sum_{i_{y}}^{i_{y}=N_{y}} \omega_{i_{y}} \frac{D_{i_{y}}}{D_{i_{y}}}=1 .
$$

The resilience metric for ICIs are obtained:

$$
\begin{gathered}
R_{m}=\frac{\int_{t_{f}}^{t_{r}} \sum_{i_{y}}^{i_{y}=N_{y}} \omega_{i_{y}} \frac{y_{i_{y}}(t)}{D_{i_{y}}} d t}{t_{r}-t_{f}}, \\
R_{r}=\frac{\int_{t_{r}}^{t_{h}} \sum_{i_{y}}^{i_{y}=N_{y}} \omega_{i_{y}} \frac{y_{i_{y}}(t)}{D_{i_{y}}} d t}{t_{h}-t_{r}}, \\
R_{t}=\frac{\int_{t_{f}}^{t_{h}} \sum_{i_{y}}^{i_{y}=N_{y}} \omega_{i_{y}} \frac{y_{i_{y}}(t)}{D_{i_{y}}} d t}{t_{h}-t_{f}} .
\end{gathered}
$$

\subsection{Global sensitivity analysis-based importance measure}

To identify the priorities of the protection and recovery strategies, we evaluate the impacts of the system parameters, i.e., failure magnitude and recovery rate of vulnerable elements by a distribution-based global sensitivity analysis (SA) method [44] that allows us to identify the most important factors affecting system resilience. Global sensitivity analysis approaches are used to analyse how the uncertainty in the output of a model can be apportioned to different sources of uncertainty in the inputs of the model [45]. Within the proposed ICIs modelling framework, the uncertainty of system parameters is the scope of the effects of the resilience activities implemented on failed elements. This approach can be used to identify the most important resilience protection and recovery activities affecting the system resilience at different stages, during a disruptive event. Choosing this global sensitivity analysis approach is because that comparing to traditional variance-based indicators and other moment independent sensitivity indicators, this approach is able to address the issues such as the presence of input variable correlations, loss of information in the distribution, etc., therefore to response to more complex 
situations, e.g., negative correlation between two repair actions due to a defined limited budget. We refer to [44] for a discussion of the advantages of this method.

The operative steps to perform such SA are given in the following. The core of the distributionbased global sensitivity analysis method is to consider the whole probability distribution of factors and to evaluate their impacts on the system outputs (e.g., the system resilience, in this paper) by an indicator with respect to the entire output distribution.

For a system model:

$$
Y=m(X), m(X): E \subseteq \mathbb{R}^{n} \rightarrow \mathbb{R}
$$

where $Y$ is the output of the model, e.g., the resilience value in our case, and $X=$ $\left(X_{1}, X_{2}, \ldots, X_{n}\right) \in R^{n}$ is the set of uncertain input variables, e.g., the failure magnitude and the recovery rate of vulnerable elements.

The importance measure (IM) for factor $X_{i}$ is:

$$
\delta_{i}=\frac{1}{2} E_{X_{i}}\left[s\left(X_{i}\right)\right]
$$

where,

$$
s\left(X_{i}\right)=\int\left|f_{Y}(y)-f_{Y \mid X_{i}}(y)\right| d y,
$$

which is the shift between the unconditional density distribution $f_{Y}(y)$ and the conditional density distribution $f_{Y \mid X_{i}}(y)$ on fixed value of $X_{i}$.

The following algorithm is carried out to obtain the IM for the factor $X_{i}$ :

Step 1. Define the ranges and probability distributions for $X_{i}, f_{i}\left(x_{i}\right)$ with $i=1, \ldots, n$.

Step 2. Compute numerically the unconditional density distribution $f_{Y}(y)$ by setting all variables as random. 
Step 3. Take a realization of $X_{i}: x_{i}=\left\{x_{i}^{1}, \ldots, x_{i}^{j}, \ldots, x_{i}^{n_{i}}\right\}, j=1, \ldots, n_{i}$, and $P\left(X_{i}=x_{i}^{j}\right)$ can be obtained from $f_{i}\left(x_{i}\right)$.

Step 4. Compute the conditional probability distribution of $X_{i}$ :

4.1) For each value in the realization of $X_{i}$, i.e., $x_{i}^{j}$ with $j=1, \ldots, n_{i}$, sample $n_{i^{\prime}}$

sets of random values of all other parameters $X_{i^{\prime}}$ with $i^{\prime} \neq i: x_{i^{\prime}}^{k} k=1, \ldots, n_{i^{\prime}}$;

4.2) Compute the $n_{i^{\prime}}$ outputs of system, i.e., $y^{j k}$ with $k=1, \ldots, n_{i^{\prime}}$, then obtain its distribution $f_{Y \mid X_{i}=x_{i}^{j}}(y)$;

4.3) Then, compute the value of $s\left(x_{i}^{j}\right)=\int\left|f_{Y}(y)-f_{Y \mid X_{i}=x_{i}^{j}}(y)\right| d y$, for $X_{i}=x_{i}^{j}$.

Step 5. Calculate the value of indicator for the factor $X_{i}: \delta_{i}=\frac{1}{2} E_{X_{i}}\left[s\left(X_{i}\right)\right]=$ $\frac{1}{2}\left[\sum_{j=1}^{n_{i}} P\left(X_{i}=x_{i}^{j}\right) s\left(x_{i}^{j}\right)\right]$.

The SA indicator can be used to rank the variables of the model. The higher the value of its SA indicator is, the higher the contribution of this variable to system output. With this method, system parameters in the ICIs model, which are associated with system protection or recovery activities, are sorted. The key resilience strategies and the most important elements are then identified in different phases of the disruptive event.

\section{Case study and results}

\subsection{Interconnected natural gas distribution network and power grid}

We consider a case study taken from [46] consisting of two interconnected CIs: a natural gas distribution network and a power grid (Fig. 4, solid and dash-dotted lines, respectively). The objective of this system is to provide the necessary amount of gas and electricity to the demand nodes. In particular, the gas distribution network supplies gas to two users, $D_{1}$ and $D_{2}$, and to two electric power generators, $E_{1}$ and $E_{2}$, that provide electricity to two users of electricity, $L_{1}$, 
and $L_{2}$.

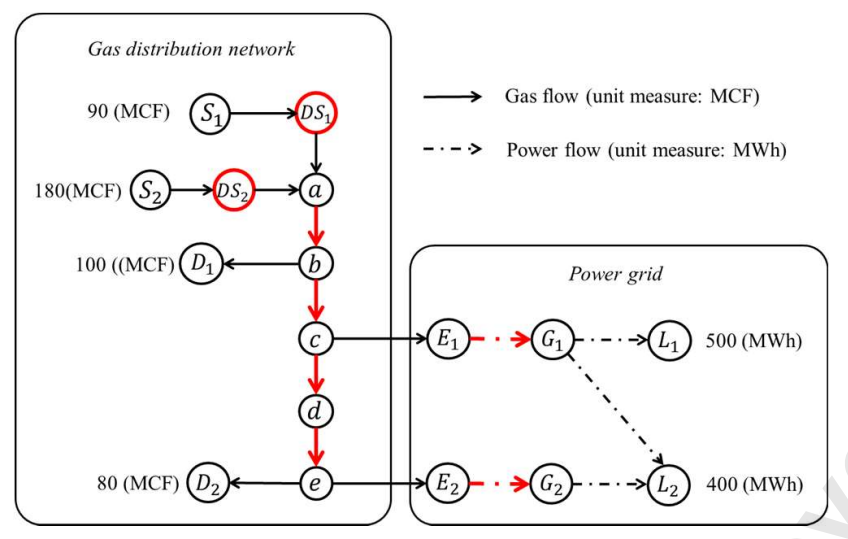

Fig. 4. Interconnected natural gas-power systems. $S$ : supplier, $D S$ : buffer/gas reservoir, $D$ : gas users. $E$ : electrical power generator, $G$ : electrical transporters, $L$ : electricity users. The vulnerable elements (nodes and links) are marked in red and bold.

The natural gas distribution network has two suppliers, $S_{1}$ and $S_{2}$, whose outputs are assumed to be equal to 90 thousand cubic feet $(\mathrm{MCF})$ and $180 \mathrm{MCF}$, respectively; two buffers (gas reservoirs), $D S_{1}$ and $D S_{2}$; five transporters $a, b, c, d$ and $e$; and two users $D_{1}$ and $D_{2}$, whose demands, $D_{D 1}$ and $D_{D 2}$, are equal to $100 \mathrm{MCF}$ and $80 \mathrm{MCF}$, respectively. The electric power network has two converters (electric power generators), $E_{1}$ and $E_{2}$, that transform gas into electricity with a constant coefficient $\beta$, where $\beta=10$ megawatt-hours (MWh) per MCF, i.e., $1 \mathrm{MCF}$ of natural gas produces $10 \mathrm{MWh}$ of electricity; two transporters, $G_{1}$ and $G_{2}$; and two users $L_{1}$ and $L_{2}$, whose demands, $D_{L 1}$ and $D_{L 2}$, are equal to $500 \mathrm{MWh}$ and $400 \mathrm{MWh}$, respectively.

TABLE 1. Ranges of uncertain system parameters

\begin{tabular}{ccc}
\hline \hline Element $i$ & Failure magnitude $F_{i}$ & Recovery rate $\mu_{i}$ \\
\hline Supplier $S_{1}$ & {$[0,90] \mathrm{MCF}$} & {$[0,1.8] \mathrm{MCF} / \mathrm{hrs}$} \\
\hline Supplier $S_{2}$ & {$[0,180] \mathrm{MCF}$} & {$[0,3.6] \mathrm{MCF} / \mathrm{hrs}$} \\
\hline Link $L_{a-b}$ & {$[0,300] \mathrm{MCF}$} & {$[0,6] \mathrm{MCF} / \mathrm{hrs}$} \\
\hline Link $L_{b-c}$ & {$[0,170] \mathrm{MCF}$} & {$[0,3.4] \mathrm{MCF} / \mathrm{hrs}$} \\
\hline Link $L_{c-d}$ & {$[0,100] \mathrm{MCF}$} & {$[0,2] \mathrm{MCF} / \mathrm{hrs}$} \\
\hline Link $L_{d-e}$ & {$[0,100] \mathrm{MCF}$} & {$[0,2] \mathrm{MCF} / \mathrm{hrs}$} \\
\hline Link $L_{E_{1}-G_{1}}$ & {$[0,800] \mathrm{MWh}$} & {$[0,16] \mathrm{MWh} / \mathrm{hrs}$} \\
\hline Link $L_{E_{2}-G_{2}}$ & {$[0,400] \mathrm{MWh}$} & {$[0,8] \mathrm{MWh} / \mathrm{hrs}$} \\
\hline \hline
\end{tabular}


Based on the data provided in [46], the vulnerable elements, i.e., the nodes and links, are marked in bold and in red as shown in Fig. 4. In the failure scenarios of the following simulation, the response time and the time horizon of a disruptive event are $H_{r}=15$ (hours) and $H_{h}$ $=85$ (hours). The failure magnitudes and recovery rates of these elements are considered as uncertain variables following uniform distributions to reflect the flexibility of the decision on system resilience strategies. The magnitude of the failure of vulnerable element $i, F_{i}$, is limited by its capacity, i.e., $F_{i}=\left[F_{i_{\text {min }}}, F_{i_{\max }}\right]=\left[0, C_{i}\right]$, where $C_{i}$ is the capacity of $i$, in units of MCF and MWh for the gas system and power grid, respectively. The recovery rate of a failed element $\mu_{i}$ can vary within $\left[\mu_{i_{\text {min }}}, \mu_{i_{\text {max }}}\right]$ where $\mu_{i_{\text {min }}}=\frac{F_{i_{\min }}}{\left(H_{h}-H_{r}\right)}$ and $\mu_{i_{\max }}=\frac{F_{i_{\max }}}{\left(H_{h}-H_{r}\right)}$, with the units $\mathrm{MCF} /$ hours and MWh/hours for the gas system and the power grid, respectively. The detailed ranges of system parameters, i.e., failure magnitudes and recovery rates of these vulnerable elements, are shown in Table 1.

\subsection{Importance measure in different phases of a disruptive event}

The evolution of system performance under the disruptive event can be evaluated within the ICIs modelling framework. The system resilience in different failure scenarios is then assessed with three resilience indicators proposed in Section 3. Subsequently, we adopt the IMs to identify the critical system elements in the mitigation and recovery phases and compare their effectiveness. The first two IM approaches are both driven by the global sensitivity analysis introduced in Section 3. The difference between them is that in the first approach, we use the indicators resilience by mitigation and resilience by recovery, and the IM taken in the recovery phase is based on the results obtained in the mitigation phase. In the second approach, total resilience is the only indicator to consider.

To test the effectiveness of the IM approaches, we design a resilience improvement scheme. A unitary increment $d v \in[0,10]$ for system parameters is considered to indicate the effects of resilience reinforcement strategies. The increment values of the system parameters rely on the priority of vulnerable elements. Based on the original level of a system parameter, e.g., the average, 
the parameter increases or reduces $5 d v$ percent if it ranks first, $4 d v$ percent if it ranks second, $3 d v$ percent if it ranks third, $2 d v$ percent if it ranks fifth, and $d v$ percent for the rest cases. Coordinating with the results from different IM approaches, this scheme is adopted in both the mitigation and recovery phases to improve the system resilience.

\subsubsection{Importance measure by SA with resilience by mitigation and resilience by recovery}

This IM approach is based on the proposed resilience metrics. With separate indicators in the mitigation and recovery phases, the global SA is performed sequentially on the mitigation phase and the restoration phase, so that the contributions of mitigation activities and recovery activities are differentiated. We consider the failure scenarios where all vulnerable elements are disturbed by a disruptive event.

In the mitigation phase, we evaluate the sensitivity of the resilience by mitigation, i.e., $R_{m}$ on the failure magnitude of vulnerable elements. The recovery rates of elements are fixed to the average levels. The SA indicator values of the failure magnitudes of the system elements are shown in Table 2 . The elements with high values of importance indicators have high priority in the mitigation phase.

TABLE 2. Importance indicators of parameters in the mitigation phase

\begin{tabular}{cccc}
\hline \hline Element $i$ & Parameter $F_{i}$ & $\mathrm{IM} \delta_{F_{i}}^{R_{m}}$ & Rank \\
\hline$S_{1}$ & $F_{S_{1}}$ & 0.0021 & 8 \\
\hline$S_{2}$ & $F_{S_{2}}$ & 0.0060 & 3 \\
\hline$L_{a-b}$ & $F_{L_{a-b}}$ & 0.0217 & 1 \\
\hline$L_{b-c}$ & $F_{L_{b-c}}$ & 0.055 & 4 \\
\hline$L_{c-d}$ & $F_{L_{c-d}}$ & 0.0031 & 6 \\
\hline$L_{d-e}$ & $F_{L_{d-e}}$ & 0.0042 & 5 \\
\hline$L_{E_{1}-G_{1}}$ & $F_{L_{E_{1}-G_{1}}}$ & 0.0120 & 2 \\
\hline$L_{E_{2}-G_{2}}$ & $F_{L_{E_{2}-G_{2}}}$ & 0.0026 & 7 \\
\hline \hline
\end{tabular}

The IM taken in the recovery phase is based on the results obtained in the mitigation phase. According to the results obtained from SA of resilience by mitigation, we improve all failure magnitude with $d v=10$. Then, we evaluate the sensitivity of the resilience by recovery, i.e., $R_{r}$ 
on the recovery rates of vulnerable elements with the update failure magnitudes. The SA indicator values in the recovery phase are shown in Table 3.

\subsubsection{Importance measure by $S A$ with total resilience}

TABLE 3. Importance measures of parameters in the recovery phase

\begin{tabular}{cccc}
\hline \hline Element $i$ & Parameter $F_{i}$ & $\mathrm{IM} \delta_{F_{i}}^{R_{r}}$ & Rank \\
\hline$S_{1}$ & $\mu_{S_{1}}$ & 0.0063 & 2 \\
\hline$S_{2}$ & $\mu_{S_{2}}$ & 0.0238 & 1 \\
\hline$L_{a-b}$ & $\mu_{L_{a-b}}$ & 0.0020 & 7 \\
\hline$L_{b-c}$ & $\mu_{L_{b-c}}$ & 0.0050 & 3 \\
\hline$L_{c-d}$ & $\mu_{L_{c-d}}$ & 0.0043 & 4 \\
\hline$L_{d-e}$ & $\mu_{L_{d-e}}$ & 0.0033 & 6 \\
\hline$L_{E_{1}-G_{1}}$ & $\mu_{L_{E_{1}-G_{1}}}$ & 0.0040 & 5 \\
\hline$L_{E_{2}-G_{2}}$ & $\mu_{L_{E_{2}-G_{2}}}$ & 0.0015 & 8 \\
\hline \hline
\end{tabular}

In this approach, we also consider the failure scenarios where all vulnerable elements are disturbed by a disruptive event. Supposing that only the total resilience $R_{t}$ is available, the global $\mathrm{SA}$ is adopted to evaluate the sensitivity of $R_{t}$ on all system parameters. The results of this IM method support the ranking of all system parameters (Column 3, Table 4). To implement the resilience improvement scheme in both the mitigation and recovery phases, we rank the failure magnitude and the recovery rate, respectively, according to the values of SA indicators. The priority order of system elements in the mitigation phase and recovery phase are then obtained (Column 4, Table 4).

\subsubsection{Importance measure by a classical method}

Then, we apply a traditional method to identify the most vulnerable elements in ICIs. Different from the previous two methods, this method combines failure occurrence probability and consequence severity of the failure on vulnerable elements to evaluate their criticality. The system performance for each failure scenario is obtained through the simulation, assuming that as a certain vulnerable element $i$ is affected by disruptions, but under the worst condition: $F_{i}=$ 
$F_{i_{\max }}$ and $\mu_{i}=\mu_{i_{\text {min }}}$

TABLE 4. Importance indicators of parameters in the mitigation phase

\begin{tabular}{cccc}
\hline \hline Parameters & IM $\delta_{F_{i}}^{R_{t}}$ & Rank & Rank in M/R phases \\
\hline$F_{S_{1}}$ & 0.0047 & 6 & 5 \\
\hline$F_{S_{2}}$ & 0.0090 & 2 & 2 \\
\hline$F_{L_{a-b}}$ & 0.0132 & 1 & 1 \\
\hline$F_{L_{b-c}}$ & 0.0050 & 5 & 4 \\
\hline$F_{L_{c-d}}$ & 0.0027 & 12 & 7 \\
\hline$F_{L_{d-e}}$ & 0.0027 & 11 & 6 \\
\hline$F_{L_{E_{1}-G_{1}}}$ & 0.0082 & 3 & 3 \\
\hline$F_{L_{E_{2}-G_{2}}}$ & 0.0025 & 15 & 8 \\
\hline$\mu_{S_{1}}$ & 0.0035 & 9 & 2 \\
\hline$\mu_{S_{2}}$ & 0.0045 & 7 & 1 \\
\hline$\mu_{L_{a-b}}$ & 0.0053 & 4 & 3 \\
\hline$\mu_{L_{b-c}}$ & 0.0037 & 8 & 6 \\
\hline$\mu_{L_{c-d}}$ & 0.0027 & 13 & 5 \\
\hline$\mu_{L_{d-e}}$ & 0.0026 & 14 & 10 \\
\hline$\mu_{L_{E_{1}-G_{1}}}$ & 0.0033 & 16 & 5 \\
\hline$\mu_{L_{E_{2}-G_{2}}}$ & 0.0025 & 16 & 5 \\
\hline \hline
\end{tabular}

The probability of initial failure occurred on $i, P E_{i}$, is computed based on the transition matrices in the semi-Markov processes [46]. The sum of the steady-state probabilities of failure modes of each vulnerable element is denoted as its failure probability, $P E_{i}$ (Column 2, Table 5). The third column in Table 5 shows the values of the total resilience $R_{t_{i}}$ in each failure scenario.

TABLE 5. Failure scenario resilience indicators

\begin{tabular}{ccc}
\hline \hline Element $i$ & $P E_{i}$ & $R_{t_{i}}$ \\
\hline Supplier $S_{1}$ & 0.3000 & 0.7522 \\
\hline Supplier $S_{2}$ & 0.0033 & 0.3819 \\
\hline Link $L_{a-b}$ & 0.0847 & 0.1077 \\
\hline Link $L_{b-c}$ & 0.0847 & 0.3294 \\
\hline Link $L_{c-d}$ & 0.0847 & 0.7113 \\
\hline Link $L_{d-e}$ & 0.0847 & 0.7016 \\
\hline Link $L_{E_{1}-G_{1}}$ & 0.0011 & 0.5796 \\
\hline \hline
\end{tabular}




\begin{tabular}{lll}
\hline \hline Link $L_{E_{2}-G_{2}}$ & 0.0013 & 0.9372 \\
\hline \hline
\end{tabular}

TABLE 6. Failure occurrence criteria

\begin{tabular}{ccc}
\hline \hline \multirow{2}{*}{ Likelihood } & $\begin{array}{c}\text { Occurrence } \\
\text { indicator } I_{i}^{o}\end{array}$ & $\begin{array}{c}\text { Score of } \\
\text { likelihood } O_{i}\end{array}$ \\
\cline { 2 - 3 } Probable & {$[0,1)$} & 10 \\
\cline { 2 - 3 } & {$[1,2)$} & 9 \\
\cline { 2 - 3 } & {$[2,3)$} & 8 \\
\hline \multirow{2}{*}{ Remote } & {$[3,4)$} & 7 \\
\cline { 2 - 3 } & {$[6,6)$} & 6 \\
\hline Extremely Remote & {$[7,8)$} & 4 \\
\cline { 2 - 3 } & {$[8,9)$} & 2 \\
\hline Extremely improbable & {$[9,+\infty)$} & 1 \\
\hline \hline
\end{tabular}

Then, we convert the failure occurrence probability and consequence severity of each vulnerable element $i$ into two criteria. The failure occurrence probability, $P E_{i}$ is used to calculate the indicator of occurrence, $I_{i}^{o}$, through $I_{i}^{o}=-\log _{10} P E_{i}$, and then the score of likelihood $O_{i}$ of the element $i$ is obtained in Table 6 . The total system resilience $R_{t_{i}}$ is transformed into the indicator of the severity of scenario $i, I_{i}^{S}$, with $I_{i}^{S}=1-R_{t_{i}}$, and then the score of likelihood $S_{i}$ can be calculated as shown in Table 7.

TABLE 7. Severity evaluation criteria

\begin{tabular}{ccc}
\hline \hline \multirow{2}{*}{$\begin{array}{c}\text { Severity } \\
\text { description }\end{array}$} & $\begin{array}{c}\text { Severity indi- } \\
\text { cator } I_{i}^{S}\end{array}$ & $\begin{array}{c}\text { Score of } \\
\text { severity } S_{i}\end{array}$ \\
\hline \multirow{2}{*}{ Catastrophic } & {$[0.9,1)$} & 10 \\
\cline { 2 - 3 } & {$[0.8,0.9)$} & 9 \\
\hline \multirow{2}{*}{ Hazardous } & {$[0.7,0.8)$} & 8 \\
\cline { 2 - 3 } & {$[0.6,0.7)$} & 7 \\
\hline \multirow{2}{*}{ Major } & {$[0.4,0.6)$} & 6 \\
\cline { 2 - 3 } & {$[0.3,0.4)$} & 5 \\
\hline \hline
\end{tabular}




\begin{tabular}{ccc}
\hline \hline & {$[0.2,0.3)$} & 3 \\
\hline \multirow{2}{*}{ Minor } & {$[0.1,0.2)$} & 2 \\
\cline { 2 - 3 } & {$[0,0.1)$} & 1 \\
\hline \multirow{2}{*}{ No effect } & 0 & 0 \\
\hline \hline
\end{tabular}

TABLE 8. Failure scenario resilience indicators

\begin{tabular}{ccc}
\hline \hline Element $i$ & $Q_{i}^{R_{t}}$ & Rank \\
\hline Supplier $S_{1}$ & 21 & 5 \\
\hline Supplier $S_{2}$ & 48 & 3 \\
\hline Link $L_{a-b}$ & 81 & 1 \\
\hline Link $L_{b-c}$ & 54 & 2 \\
\hline Link $L_{c-d}$ & 18 & 6 \\
\hline Link $L_{d-e}$ & 18 & 6 \\
\hline Link $L_{E_{1}-G_{1}}$ & 32 & 4 \\
\hline Link $L_{E_{2}-G_{2}}$ & 8 & 7 \\
\hline \hline
\end{tabular}

Based on the scores of failure likelihood and the scores of consequence severity for the vulnerable elements, we calculate their priority indicators, $Q_{i}^{R_{t}}=S_{i} \cdot O_{i}$. The results are shown in Table 8. With this classical method, the rank of vulnerable elements is obtained. The four most important elements are: $\operatorname{link} L_{a-b}$, link $L_{b-c}$, supplier $S_{2}$ and link $L_{E_{1}-G_{1}}$, and this order will be considered in the resilience improvement scheme in both mitigation and recovery phases.

\subsection{Comparison of results}

We have obtained the ranks of vulnerable system elements by three IM approaches: 1) global SA-based IM considering mitigation and recovery aspects separately, 2) global SA-based IM with total resilience indicator, and 3) a classical method. We are interested in comparing the effects of their ranking results.

TABLE 9. The rank of system parameters in the mitigation phase

\begin{tabular}{cccc}
\hline \hline Rank & Method 1 & Method 2 & Method 3 \\
\hline 1 & $L_{a-b}$ & $L_{a-b}$ & $L_{a-b}$ \\
\hline 2 & $L_{E_{1}-G_{1}}$ & $L_{E_{1}-G_{1}}$ & $L_{b-c}$ \\
\hline \hline
\end{tabular}




\begin{tabular}{cccc}
\hline \hline 3 & $S_{2}$ & $S_{2}$ & $S_{2}$ \\
\hline 4 & $L_{b-c}$ & $L_{b-c}$ & $L_{E_{1}-G_{1}}$ \\
\hline 5 & $L_{d-e}$ & $S_{1}$ & $S_{1}$ \\
\hline 6 & $L_{c-d}$ & $L_{d-e}$ & $L_{c-d} L_{d-e}$ \\
\hline 7 & $L_{E_{2}-G_{2}}$ & $L_{c-d}$ & $L_{E_{2}-G_{2}}$ \\
\hline 8 & $S_{1}$ & $L_{E_{2}-G_{2}}$ & \\
\hline \hline
\end{tabular}

TABLE 10. The rank of system parameters in the recovery phase

\begin{tabular}{cccc}
\hline \hline Rank & Method 1 & Method 2 & Method 3 \\
\hline 1 & $S_{2}$ & $L_{a-b}$ & $L_{a-b}$ \\
\hline 2 & $L_{b-c}$ & $S_{2}$ & $L_{b-c}$ \\
\hline 3 & $L_{c-d}$ & $L_{b-c}$ & $S_{2}$ \\
\hline 4 & $S_{1}$ & $L_{E_{1}-G_{1}}$ & $L_{E_{1}-G_{1}}$ \\
\hline 5 & $L_{E_{1}-G_{1}}$ & $S_{1}$ & $S_{1}$ \\
\hline 6 & $L_{d-e}$ & $L_{c-d}$ & $L_{c-d} L_{d-e}$ \\
\hline 7 & $L_{a-b}$ & $L_{d-e}$ & $L_{E_{2}-G_{2}}$ \\
\hline 8 & $L_{E_{2}-G_{2}}$ & $L_{E_{2}-G_{2}}$ & \\
\hline \hline
\end{tabular}

The ranking order indicating the criticality of the vulnerable elements in the mitigation phase and the recovery phase are shown in Table 9 and Table 10, respectively. The results provided by method 1 show that the priority of system elements in the recovery phase is different from that in the mitigation phase. In the mitigation phase, the rank order obtained from method 1 is quite similar to method 2. All three approaches confirm the criticality of the link $L_{a-b}$. In the recovery phase, their results from the three methods are dissimilar.

To test the effects of three sorting methods, we consider improving the system resilience according to the sorting results from the three IM methods, under the same original failure scenario, i.e., failure magnitudes and recovery rates of the elements are equal to their average levels. The resilience improvement schema is applied: failure magnitudes and recovery rates of the elements are reduced or increased according to the ranking results in the mitigation phase and recovery phase, respectively. 
Fig. 5 shows the evolvement of the curve of system resilience, $R_{t}$, with the increasing increment of system parameters, $d v$. Notice that the y-axis representing $R_{t}$ is in the range of $[0,1]$ by its definition given in Eq. (21). When the increment is small, the classical method produces a better effect. But with the increase of increment, the first IM method, i.e., the method based on the proposed resilience indicators, shows its effectiveness.

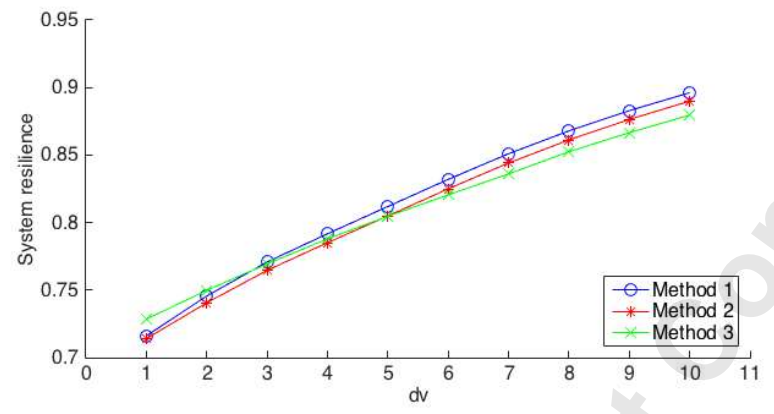

Fig. 5. Improvement of system resilience with different levels of increment $d v$

More precisely, we take a look at the case where $d v=10$ to compare system performance of ICIs after different resilience improvement strategies are taken. As shown in Fig. 6, the lowest value of system performance with method 1 and method 2 is the same, as they have similar ranking results in the mitigation phase. With method 3 , the failure propagation process is longer and the lowest value reached by the system performance curve is lower than others. In the recovery phase, the improved system performance with method 1 is the first to recovers back to the nominal value.

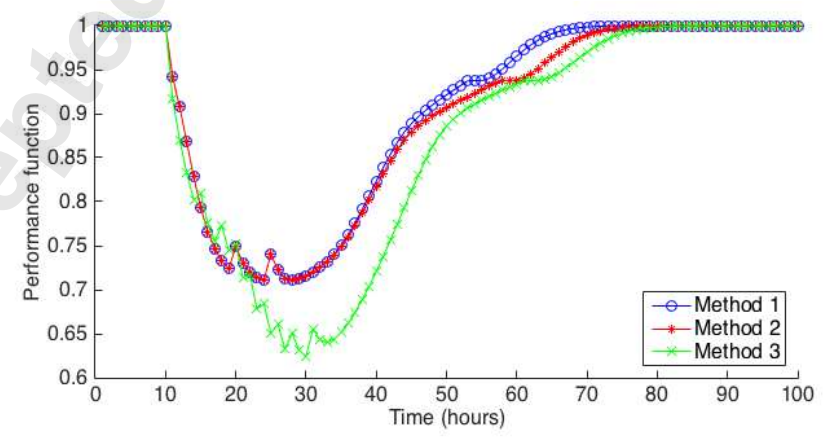

Fig. 6. The evolvement of system performance with three methods, $d v=10$

Comparing to the other two approaches, the IM approach with the proposed resilience metric is 
more effective. That is because the proposed resilience indicators allow us to obtain updated priority of system elements in mitigation and recovery phases.

The limitation of the IM approach with the proposed resilience metric is the relatively high computational cost. To calculate SA indicator of each system parameter in any of the two phases requires $10^{6}$ iterations. Therefore, the computational cost of method 1 is two times of method 2 and is much more expensive than method 3.

\section{Conclusion}

This paper proposed a generic system resilience metric, which evaluates separately the two aspects of system resilience, i.e., the capacity of resistance gained from protective measures implemented and the capacity of recovery owing to the restoration actions. By taking into account the characteristics of ICIs, we evaluate the system performance in terms of users' satisfaction within a control-based dynamic modelling framework. Then, we apply the proposed resilience metric to measure the criticality of system elements from the perspective of their contribution to system resilience in the mitigation and recovery phases. A global SA approach is used to evaluate the level of dependencies of the system resilience on system parameters associating with the system elements.

Three importance measure (IM) approaches are performed on a case study composing of a natural gas distribution network and a power grid. The first one is a global SA-based IM with resilience by mitigation and resilience by recovery indicators, i.e., the proposed resilience metric. The second one is also global SA-driven, but only considers the total system resilience. The third one is a classical method combining the failure occurrence probability and consequence severity in the worst failure scenario of each vulnerable element. We obtained the ranks of system parameters in different phases of a disruptive event and then conclude the priorities of vulnerable ele-

ments which should be protected and recovered. A system resilience improvement scheme is considered to compare the effectiveness of three IM methods. Comparing to the other two approaches, the IM approach with the proposed resilience metric shows higher effectiveness. 
Note that the resilience assessment approaches in literature is not able to quantitatively differentiate the contributions of the mitigation and recovery aspects, so that cannot provide decision-makers the separate priority of system elements in different phases. Instead, the proposed multiple resilience indicators and the corresponding IM approach provide more insights to decision-makers to increase the effectiveness of system resilience improvement strategies. Moreover, the generic resilience metric is also applicable to a variety of domains and many situations, such as system resilience design, system resilience improvement, and system resilience examination.

The proposed IM approach is computationally costly compared to existing methods. Nevertheless, it should be mentioned that this is not necessarily a real concern due to the significant advancement in computing power. Actually, the proposed approach can be applied to both real time tactical emergency management and long-term strategic planning, for which computation is often done offline. For long-term strategic planning, historical failure data can be fed into the proposed approach for identifying the statistically most vulnerable and critical elements in ICIs, for protection and upgrading. Also, our approach is able to support pre-disruption preparedness activities when the most significant characteristics of the emergent hazard, and the associated failure scenarios of the system, can be postulated (e.g., for tropical storms and hurricanes, flooding, blizzards, etc.)

In real emergency scenarios, system operation parameters (such as the electricity and gas demand) might be highly variable and uncertain and affect the system performance quantification. This should be taken into account in the robust application of the proposed method. Last but not least, this study has not considered the cost associated with the resilience reinforcement strategies, which requires detailed models of the protection and restoration actions of system components. Such developments will be investigated in future works.

\section{References}

[1] Kröger W, Zio E. Vulnerable Systems. London: Springer London, 2011. Epub ahead of print 2011. DOI: 10.1007/978-0-85729-655-9. 
[2] Zio E. Challenges in the vulnerability and risk analysis of critical infrastructures. Reliab Eng Syst Saf 2016; 152: 137-150.

[3] Zio E. Reliability engineering: Old problems and new challenges. Reliab Eng 2009; 94: $125-141$

[4] Buldyrev S V., Parshani R, Paul G, et al. Catastrophic cascade of failures in interdependent networks. Nature 2010; 464: 1025-1028.

[5] Fang Y, Zio E. Game-Theoretic Decision Making for the Resilience of Interdependent Infrastructures Exposed to Disruptions. 2019; 97-114.

[6] Fang Y-P, Zio E. An adaptive robust framework for the optimization of the resilience of interdependent infrastructures under natural hazards. Eur J Oper Res; 276. Epub ahead of print 2019. DOI: 10.1016/j.ejor.2019.01.052.

[7] Yu J-Z, Baroud H. Modeling Uncertain and Dynamic Interdependencies of Infrastructure Systems Using Stochastic Block Models. ASCE-ASME J Risk Uncert Engrg Sys Part B Mech Engrg; 6.

[8] Ayyub BM. Systems resilience for multihazard environments: Definition, metrics, and valuation for decision making. Risk Anal 2014; 34: 340-355.

[9] Salomon J, Broggi M, Kruse S, et al. Resilience Decision-Making For Complex Systems. ASCE-ASME J Risk Uncert Engrg Sys Part B Mech Engrg; 6.

[10] Ayyub BM, Pantelous AA, Shao J. Toward Resilience to Nuclear Accidents: Financing Nuclear Liabilities via Catastrophe Risk Bonds. ASCE-ASME J Risk Uncert Engrg Sys Part B Mech Engrg; 2.

[11] Hosseini S, Barker K, Ramirez-Marquez JE. A review of definitions and measures of system resilience. Reliab Eng Syst Saf 2016; 145: 47-61.

[12] Henry D, Ramirez-Marquez JE. Generic metrics and quantitative approaches for system resilience as a function of time. Reliab Eng Syst Saf 2012; 99: 114-122.

[13] Yodo N, Wang P. Engineering Resilience Quantification and System Design Implications: A Literature Survey. J Mech Des 2016; 138: 111408.

[14] Jin C, Li R, Kang R, et al. Analysis and comparison of three measures for system 
resilience. In: Risk,Reliabiltiy and Safety: Innovating Theory and Practice. Taylor \& Francis Group, London, 2017, pp. 2145-2151.

[15] Bruneau M, Chang SE, Eguchi RT, et al. A Framework to Quantitatively Assess and Enhance the Seismic Resilience of Communities. Earthq Spectra 2003; 19: 733-752.

[16] Ouyang M, Dueñas-Osorio L, Min X. A three-stage resilience analysis framework for urban infrastructure systems. Struct Saf 2012; 36-37: 23-31.

[17] Fang Y-P, Pedroni N, Zio E. Resilience-Based Component Importance Measures for Critical Infrastructure Network Systems. IEEE Trans Reliab 2016; 65: 502-512.

[18] Nan C, Sansavini G. A quantitative method for assessing resilience of interdependent infrastructures. Reliab Eng Syst Saf 2017; 157: 35-53.

[19] Kong J, Simonovic SP. Probabilistic multiple hazard resilience model of an interdependent infrastructure system. Risk Anal 2019; 39: 1843-1863.

[20] Francis R, Bekera B. A metric and frameworks for resilience analysis of engineered and infrastructure systems. Reliab Eng Syst Saf 2014; 121: 90-103.

[21] Fisher. et.al. Constructing a Resilience Index for the Enhanced Critical Infrastructure Protection Program. Argonne Natl Lab 2013; 53: 1689-1699.

[22] Westmark VR. A definition for information system survivability. 37th Annu Hawaii Int Conf Syst Sci 2004 Proc 2004; 00: 1-10.

[23] Jackson S, Ferris TLJ. Resilience principles for engineered systems. Syst Eng 2013; 16: $152-164$.

[24] Uday P, Marais K. Designing Resilient Systems-of-Systems: A Survey of Metrics, Methods, and Challenges. Syst Eng 2015; 18: 491-510.

[25] Zobel CW. Representing perceived tradeoffs in defining disaster resilience. Decis Support Syst 2011; 50: 394-403.

[26] Rose A. Economic resilience to natural and man-made disasters: Multidisciplinary origins and contextual dimensions. Environ Hazards 2007; 7: 383-398.

[27] Liu X, Ferrario E, Zio E. Resilience analysis framework for interconnected critical infrastructures. ASCE-ASME J Risk Uncert Engrg Sys, Part B Mech Engrg 2017; 3: 1- 
10.

[28] Galbusera L, Ntalampiras S, Azzini I, et al. Resilience assessment and optimization methodology for critical infrastructure. 2013.

[29] Liu Y-Y, Slotine J-J, Barabási A-L. Controllability of complex networks. Nature 2011; 473: $167-173$.

[30] Muller G. Fuzzy architecture assessment for critical infrastructure resilience. Procedia Comput Sci 2012; 12: 367-372.

[31] Nair R, Avetisyan H, Miller-Hooks E. Resilience Framework for Ports and Other Intermodal Components. Transp Res Rec J Transp Res Board 2010; 2166: 54-65.

[32] Prodan I, Zio E. A model predictive control framework for reliable microgrid energy management. Int J Electr Power Energy Syst 2014; 61: 399-409.

[33] Camacho EF, Bordons Alba C. Model Predictive Control. Springer Science \& Business Media, 2013.

[34] Findeisen R, Allgöwer F. An introduction to nonlinear model predictive control. In: 21st Benelux meeting on systems and control. Technische Universiteit Eindhoven Veldhoven Eindhoven, The Netherlands, 2002, pp. 119-141.

[35] Alessio A, Bemporad A. A survey on explicit model predictive control. In: Nonlinear model predictive control. Springer, 2009, pp. 345-369.

[36] Bemporad A, Morari M. Robust model predictive control: A survey. In: Robustness in identification and control. Springer, 1999, pp. 207-226.

[37] Mayne DQ. Model predictive control: Recent developments and future promise. Automatica 2014; 50: 2967-2986.

[38] Cimellaro GP, Reinhorn AM, Bruneau M. Framework for analytical quantification of disaster resilience. Eng Struct 2010; 32: 3639-3649.

[39] Fang Y-P, Pedroni N, Zio E. Comparing Network-Centric and Power Flow Models for the Optimal Allocation of Link Capacities in a Cascade-Resilient Power Transmission Network. IEEE Syst J 2014; 1-12.

[40] Dessavre DG, Ramirez-Marquez JE, Barker K. Multidimensional approach to complex 
system resilience analysis. Reliab Eng Syst Saf 2016; 149: 34-43.

[41] Ganin AA, Massaro E, Gutfraind A, et al. Operational resilience: concepts, design and analysis. Sci Rep 2016; 6: 1-12.

[42] Ayyub BM. Practical resilience metrics for planning, design, and decision making. ASCEASME J Risk Uncertain Eng Syst Part A Civ Eng 2015; 1: 4015008.

[43] Cimellaro GP, Reinhorn AM, Bruneau M. Seismic resilience of a hospital system. Struct Infrastruct Eng 2010; 6: 127-144.

[44] Borgonovo E. A new uncertainty importance measure. Reliab Eng Syst Saf 2007; 92: 771784 .

[45] Saltelli A. Global Sensitivity Analysis: An Introduction. Sensit Anal Model Output 2005; $27-43$.

[46] Nozick LK, Turnquist MA, Jones DA, et al. Assessing the performance of interdependent infrastructures and optimising investments. Int J Crit Infrastructures 2005; 1: 144-154. 